\title{
Multifrequency gravitational wave background from continuous sources
}

\author{
C Sivaram ${ }^{1}$ and Arun Kenath ${ }^{2, *}$ \\ 1 Indian Institute of Astrophysics, Bangalore, India; sivaram@iiap.res.in \\ 2 Christ Junior College and Department of Physics and Electronics, CHRIST (Deemed to be University), \\ Bangalore, India; kenath.arun@cjc.christcollege.edu \\ * Correspondence: kenath.arun@cjc.christcollege.edu; Tel.: +91-80-4012-9292
}

\begin{abstract}
Gravitational waves have been detected in the past few years from several transient events such as merging stellar mass black holes, binary neutron stars, etc. These waves have frequencies in a band ranging from a few hundred hertz to around a kilohertz to which LIGO type instruments are sensitive. LISA would be sensitive to much lower range of frequencies from SMBH mergers. Apart from these cataclysmic burst events, there are innumerable sources of radiation which are continuously emitting gravitational waves of all frequencies. These include a whole mass range of compact binary and isolated compact objects as well as close planetary stellar entities. In this work, quantitative estimates are made of the gravitational wave background produced in typical frequency ranges from such sources emitting over a Hubble time and the fluctuations in the $h$ values measured in the usual devices. Also estimates are made of the high frequency thermal background gravitational radiation from hot stellar interiors and newly formed compact objects.
\end{abstract}

Keywords: gravitational waves; neutron star mergers; black hole mergers; primordial black holes; continuous gravitational waves; thermal gravitational waves; detection of gravitational waves

\section{Introduction}

Gravitational waves (GW) as predicted by Einstein over a century ago, were first detected by LIGO in 2016 [1]. The waves came from two black holes circling closer and closer to each other till they finally collided and coalesced. Most of the radiation was released in the final orbit, which had a period of about a millisecond, with the wave frequency from $0.6-1.2 \mathrm{kHz}$, typical of such stellar events. About three solar masses were converted to energy of gravitational waves, i.e. $\sim 5 \times 10^{54} \mathrm{erg}$. LIGO has detected several such events of black hole mergers since. Apart from black hole mergers, in 2017, LIGO detected gravitational waves from the collision of two neutron stars. Unlike the black hole mergers which are only detectable gravitationally, this event (GW170817) was also detected electromagnetically [2].

These are long wavelength, low frequency gravitational waves. Future gravitational wave detectors like LISA would be sensitive to much longer wavelengths and lower fluxes [3]. However compact stellar objects can generate high frequency thermal gravitational radiation, which in the case of hot neutron stars can be high. Also white dwarfs and main-sequence stars can generate such radiation from plasma-Coulomb collisions. Gamma ray bursts could also be sources of such radiation, as also the terminal stages of evaporating black holes $[4,5]$.

Here a study is made of the thermal gravitational wave emission from all of the above sources, and the background flux is estimated. The earliest phases of the Universe close to the Planck scale would also leave remnant thermal gravitational waves. The integrated thermal gravitational flux as the Universe expands is also estimated and compared with that from all the discrete sources discussed above. Possible schemes to detect such sources of high frequency thermal gravitational radiation are discussed and the physical principles involved are elaborated. 


\section{Gravitational waves from merger of compact binary objects}

Gravitational wave detectors like LIGO are hunting for waves from compact binary inspirals, which are produced by orbiting pairs of massive, compact objects like neutron stars (NS) and black holes $(\mathrm{BH})$. Gravitational waves detected so far by LIGO fall in this category. These compact binary systems include binary neutron stars, binary black holes, and neutron star - black hole binary.

It is expected that there should be about a billion neutron stars in the Milky Way. Of the total of $\sim 10^{9}$ neutron stars in the galaxy, about half $\left(5 \times 10^{8}\right)$ will be part of NS binaries [6]. The two neutron stars orbiting each other closely emit gravitational radiation and spiral inward. Their merger leads to the formation of either a more massive neutron star, or a black hole (depending on whether the mass of the remnant exceeds the Tolman-Oppenheimer-Volkoff limit). The power radiated through GW for an equal mass binary system, each of mass $M$, separated by a distance $a$ (circular orbit), is given by [7]:

where the frequency of gravitational waves is:

$$
P=\frac{32}{5} \frac{G}{c^{5}} M^{2} a^{4} \omega^{6}
$$

$$
\omega \approx\left(\frac{G M}{a^{3}}\right)^{1 / 2}
$$

The merger time of the equal mass binary system is given by:

$$
t_{\text {merger }}=\frac{5}{64} \frac{c^{5}}{G^{3}} \frac{a^{4}}{M^{3}}
$$

i.e. the merger time is related to the mass and separation as, $t_{\text {merger }} \propto \frac{a^{4}}{M^{3}}$.

For the merger time to be of the order of the Hubble time, i.e. $t_{\text {merger }}=t_{H}\left(\approx 4.35 \times 10^{17} \mathrm{~s}\right)$, we have following equation (3), $t_{H} M^{3}=$ constant $\times a^{4}$. For instance, an equal mass binary, each $2 M_{\odot}$, would merge in Hubble time, if their initial separation is $\approx 2.5 \times 10^{11} \mathrm{~cm}$. The maximum initial separation $\left(a_{i}\right)$ of the equal mass binary of different mass range to merge within the Hubble time is given in figure 1.

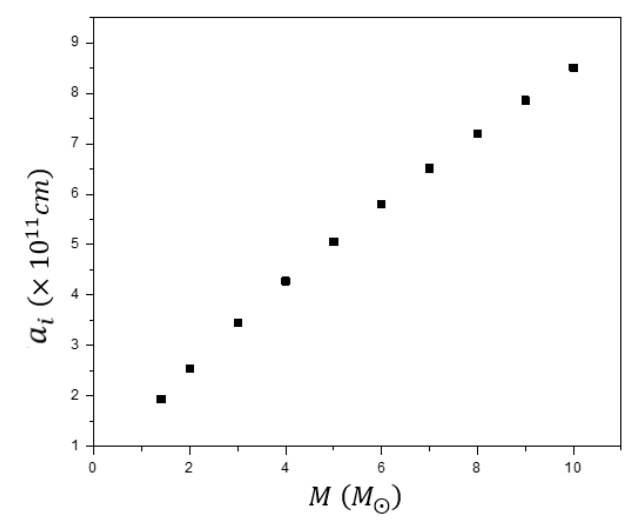

Figure 1. Initial separation for various mass for $t_{\text {merger }}=t_{H}$.

From this we can express the separation in terms of the mass as:

$$
a=\left(\frac{t_{H} M^{3}}{\text { constant }}\right)^{1 / 4}=\text { constant }^{\prime} \times M^{3 / 4}
$$

From equations (4) and (2) we have the frequency of GW in terms of the mass $M$ as:

$$
\omega=\text { constant }^{\prime \prime} \times M^{-5 / 8}
$$

With the constants, this scaling of $\omega$ with mass $M$ is give as:

$$
\omega=2.7 \times 10^{-4} \mathrm{~Hz}\left(\frac{M}{M_{\odot}}\right)^{-5 / 8}
$$

From equations (4) and (5), we have $a^{4} \propto M^{3}$ and $\omega^{6} \propto M^{-15} / 4$ respectively. These along with equation (1), gives the mass dependence of power, $P$ as, $P \propto M^{5 / 4}$. With the constants this scaling is given as:

$$
P=1.5 \times 10^{31} \mathrm{erg} / \mathrm{s}\left(\frac{M}{M_{\odot}}\right)^{5 / 4}
$$


Figure 2(a) gives the variation of frequency $(\omega)$ of GW with mass $(M)$ and figure 2(b) gives the variation of power $(P)$ emitted in GW with mass $(M)$. The total energy emitted over the Hubble time $\left(t_{H}\right)$ is $E_{H}=t_{H} \times P$. This will give a background flux which could affect gravitational waves measured from distant bursts. These values for varying masses of the equal mass binary black holes are tabulated in table 1. These values of frequency and power are those associated with the initial stages of the separation of the equal mass binaries. They evolve over time as the separation decreases, with both $\omega$ and $P$ increasing with decreasing separation. The frequency detected by gravitational wave detectors, like LIGO, is that during the merger as the separation, $a \approx 2 \times$ radius of the NS, or $2 \times$ Schwarzschild radius in the case of BHs.

Table 1. Variation with BH mass of frequency, power, and total energy over Hubble time emitted in GW.

\begin{tabular}{cccc}
\hline $\boldsymbol{M}\left(\boldsymbol{M}_{\odot}\right)$ & $\boldsymbol{\omega}(\boldsymbol{H z})$ & $\boldsymbol{P}(\boldsymbol{e r g} / \boldsymbol{s})$ & $\boldsymbol{E}_{\boldsymbol{H}}(\boldsymbol{e r g})$ \\
\hline 5 & $9.87 \times 10^{-5}$ & $1.12 \times 10^{32}$ & $4.87 \times 10^{49}$ \\
6 & $8.81 \times 10^{-5}$ & $1.41 \times 10^{32}$ & $6.13 \times 10^{49}$ \\
7 & $8.00 \times 10^{-5}$ & $1.71 \times 10^{32}$ & $7.44 \times 10^{49}$ \\
8 & $7.36 \times 10^{-5}$ & $2.02 \times 10^{32}$ & $8.79 \times 10^{49}$ \\
10 & $6.40 \times 10^{-5}$ & $2.67 \times 10^{32}$ & $1.16 \times 10^{50}$ \\
15 & $4.97 \times 10^{-5}$ & $4.43 \times 10^{32}$ & $1.93 \times 10^{50}$ \\
20 & $4.15 \times 10^{-5}$ & $6.34 \times 10^{32}$ & $2.76 \times 10^{50}$ \\
25 & $3.61 \times 10^{-5}$ & $8.38 \times 10^{32}$ & $3.64 \times 10^{50}$ \\
30 & $3.22 \times 10^{-5}$ & $1.05 \times 10^{33}$ & $4.57 \times 10^{50}$ \\
40 & $2.69 \times 10^{-5}$ & $1.51 \times 10^{33}$ & $6.57 \times 10^{50}$ \\
50 & $2.34 \times 10^{-5}$ & $1.99 \times 10^{33}$ & $8.66 \times 10^{50}$ \\
60 & $2.09 \times 10^{-5}$ & $2.50 \times 10^{33}$ & $1.09 \times 10^{51}$ \\
70 & $1.90 \times 10^{-5}$ & $3.04 \times 10^{33}$ & $1.32 \times 10^{51}$ \\
80 & $1.75 \times 10^{-5}$ & $3.59 \times 10^{33}$ & $1.56 \times 10^{51}$ \\
90 & $1.62 \times 10^{-5}$ & $4.16 \times 10^{33}$ & $1.81 \times 10^{51}$ \\
100 & $1.52 \times 10^{-5}$ & $4.74 \times 10^{33}$ & $2.06 \times 10^{51}$ \\
\hline
\end{tabular}

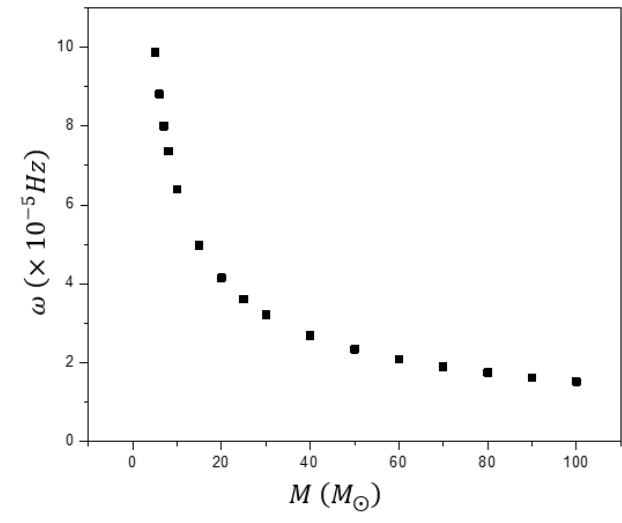

(a)

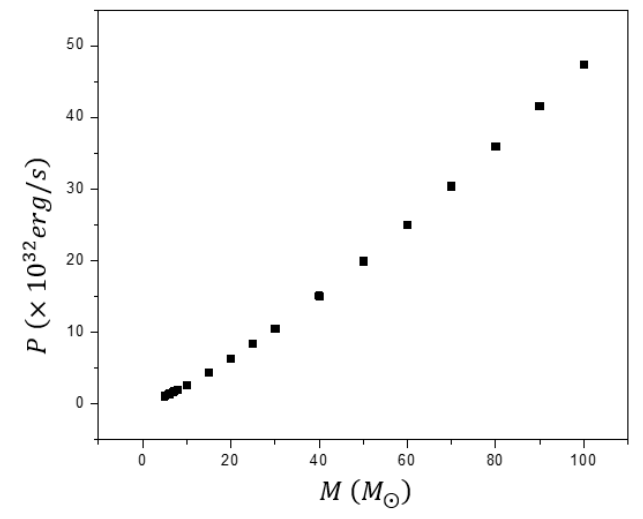

(b)

Figure 2. Variation with mass of the binary components in the: (a) frequency of GW; (b) power emitted in GW.

As the binary components continue to approach each other, the frequency increases, culminating with the chirp frequency as they ultimately merge which is the one measured by GW detectors. The rate of change of the frequency with decreasing distance between the binary components is given by: 


$$
\frac{d \omega}{d a}=\left(\frac{9 G M}{2 a^{5}}\right)^{1 / 2}
$$

The variation in frequency - for binary systems with different masses - with respect to decreasing distance (as they approach) is plotted in figure 3(a). As the binary components continue to inspiral, the power emitted due to gravitational radiation increases, and the corresponding change in power is:

$$
\frac{d P}{d a}=\frac{32 G^{4} M^{5}}{c^{5} a^{6}}
$$

The variation in power with respect to decreasing distance is plotted in figure 3(b), for binary systems with different mass.

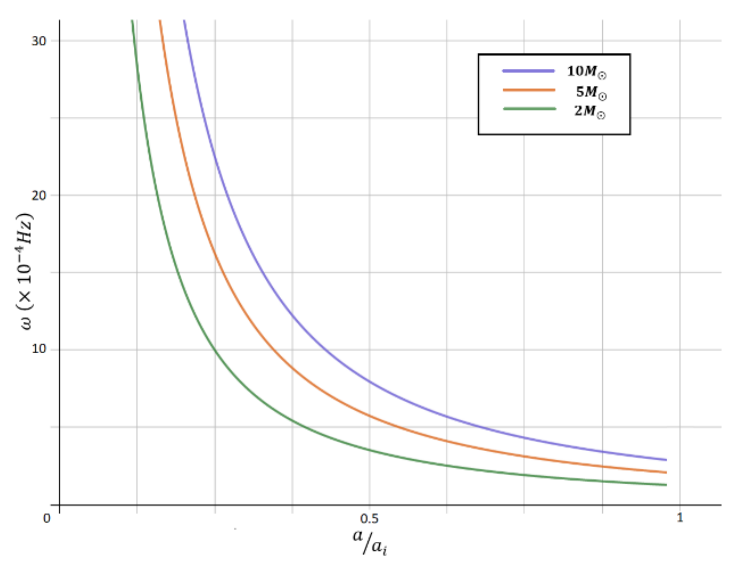

(a)

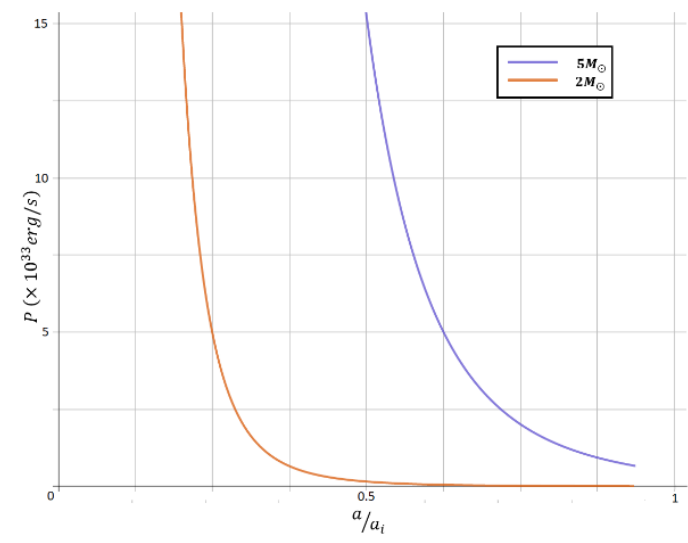

(b)

Figure 3. Variation with decreasing distance between equal mass binaries (of different masses) in the: (a) frequency; (b) power radiated.

The total power radiated during the merger can be obtained by integrating equation (9) from the initial separation to that at the merger ( $\approx 2 \times$ radius of the NS, or $2 \times$ Schwarzschild radius for BHs). For a $2 M_{\odot}$ NS binary, the total energy emitted in GW over the merger time is then given as:

$$
E=\frac{2.7 \times 10^{54}}{t(\text { in s })^{1 / 4}}
$$

For a merger time of about a billion years, the total energy emitted is $E \approx 10^{51} \mathrm{erg}$. With one NS merger per galaxy in $10^{5}$ years, the total number of mergers in the whole Universe (with $\sim 10^{11}$ galaxies) is $\sim 10^{6}$ per year. Then in the Hubble time, total mergers that have already taken place would be $\sim 10^{16}$. Each of these mergers would release an energy of $\sim 10^{52} \operatorname{ergs}\left(=G M^{2} / R\right.$, the binding energy of the NS, where $R$ is the radius of the NS) in GW. This then implies that a total energy of $\sim 10^{68}$ ergs is released in GW. This is spread over the Hubble volume of $2 \pi^{2} R_{H}^{3} \approx 10^{85} c c$, hence the energy density is $\sim 10^{-17} \mathrm{ergs} / \mathrm{cc}$ and the corresponding flux on Earth is $\sim 10^{-9} \mathrm{ergs} / \mathrm{cm}^{2} / \mathrm{s}$ in the frequency range of $100 \mathrm{~Hz}-1 \mathrm{kHz}$ (the chirp frequency detected by the GW detectors at the final stages of the merger). The flux of gravitational waves is related to the strain as:

$$
f=\frac{c^{3}}{32 \pi G} \omega^{2} h^{2}
$$

For frequency of $100 \mathrm{~Hz}$, the strain will be: $h \approx 10^{-24}$. This is the strain due to the background flux, and it is about one order lesser than the sensitivity of LIGO, which is $\approx 10^{-23}$.

In the case of BHs, a $20-30 M_{\odot} \mathrm{BH}$ is formed by the collapse of a $50 M_{\odot}$ progenitor star. On average a massive star collapses every second in the Universe. So in the Hubble time, total of $\sim 10^{17}$ such collapses will occur. The number of stars from 20 to $50 M_{\odot}$ mass range is given by (using the usual Salpeter mass function):

$$
N=\int_{20 M_{\odot}}^{50 M_{\odot}} \frac{k d M}{M^{2.5}}
$$

where, $k \sim 1$ star $/\left(p c^{3} M_{\odot}\right)$, this works out to be, $N=0.02 \operatorname{star} /\left(p c^{3} M_{\odot}\right)$.

Stellar mass BH mergers are estimated to occur once every $200-300$ seconds in the Universe. So the total number of such mergers in the Hubble time will be $\sim 10^{15}$. The energy flux is comparable to NS 
mergers. The energy released is 10 - 100 times more than that in the case of NS mergers, but the frequency is 100 times lower, hence the background strain is comparable.

In the case of mergers of SMBH, the frequency is very low, but the energies are much higher, so only LISA can detect the corresponding background strain. For example, the binary system OJ 287 consists of two orbiting supermassive black holes about a Gpc away. The mass of the primary black hole is deduced to be 18 billion solar mass, and the secondary black hole of OJ 287 has a mass of 150 million solar mass. For the orbital period of 12 years, the orbit separation is $\sim 10^{4} \mathrm{AU}$. The energy released as gravitational radiation when the merger occurs would be $\sim 10^{62} \mathrm{erg}$. Even at Gpc distance the strain on a gravitational wave detector would be, $h \approx 10^{-16}$, with a typical frequency $\sim 5-10$ microhertz. This would be a very 'bright' signal for LISA (which has a threshold of $\sim 10^{-23}$ ) [8].

\section{Thermal gravitational waves: Sources and background}

Apart from mergers of binary compact objects, compact stellar objects can generate high frequency $\left(10^{16}-10^{21} \mathrm{~Hz}\right)$ thermal gravitational radiation. In the case of hot neutron stars, these thermal GW can be high. Main-sequence stars can generate such thermal gravitational radiation from plasma-Coulomb collisions, as also white dwarfs. Apart from these stellar and stellar remnants, gamma ray bursts could also be a source of thermal GW. Another possible source of such thermal GW are the terminal stages of evaporating black holes.

\subsection{Thermal gravitational waves from hot stellar cores and compact stellar remnants}

Due to Coulomb collisions in the core of the stars, thermal gravitational waves can be generated. These thermal gravitational waves can arise in white dwarfs and neutron stars due to the fermion collisions in the dense degenerate Fermi gas.

If $n_{1}, n_{2}$ are the number densities of gas particles undergoing collision with a $\frac{d \sigma_{12}}{d \Omega}$ differential scattering cross-section, with relative velocity $v_{12}$ and reduced mass $\mu_{12}$, then the power per unit volume per unit frequency interval is given by the quadrupole formula as [9]:

$$
\dot{E}=\left(\frac{32 G}{5 c^{5}} \mu_{12}^{2} n_{1} n_{2} v_{12}^{5} \sum \frac{d \sigma_{12}}{d \Omega} \sin ^{2} \theta\right) V v
$$

where, $V$ is the volume of the stellar core and $v=\frac{k T_{c o r e}}{h} \approx 10^{17} \mathrm{~Hz}$ is the frequency corresponding to the core temperature of the star of $T_{\text {core }} \approx 10^{7} \mathrm{~K}$, and the corresponding velocity of the particles, $v_{12} \approx$ $8 \times 10^{7} \mathrm{~cm} / \mathrm{s}$. The typical number density, $n_{1}=n_{2}=\rho / m_{P} \approx 10^{26} \mathrm{~cm}^{-3}$, and volume of the stellar core, $V \approx 10^{33} \mathrm{~cm}^{3}$. And for a main sequence star [10],

$$
\sum \frac{d \sigma_{12}}{d \Omega} \sin ^{2} \theta=\frac{e^{4}}{\mu_{12}^{2} v_{12}^{4}} \approx 5 \times 10^{-24} \mathrm{~cm}^{2}
$$

These values give the power of thermal gravitational waves emitted as, $\dot{E} \approx 10^{16} \mathrm{erg} / \mathrm{s}$, at a frequency of, $v \approx 10^{17} \mathrm{~Hz}$. The flux of thermal gravitational waves from the Sun, received at Earth is of the order of half a watt.

Considering all the $10^{11}$ stars in all the 200 billion galaxies, the power associated with the thermal gravitational waves from all these stars is of the order of, $\dot{E} \approx 10^{38} \mathrm{erg} / \mathrm{s}$. The total energy emitted due to the thermal gravitational waves by these stars over their average life span of $\approx 3 \times 10^{17} s$ is, $E \approx$ $3 \times 10^{55} \mathrm{erg} / \mathrm{s}$.

In the case of white dwarfs (WD), the number density is of the order of, $n_{1}=n_{2} \approx 10^{31} \mathrm{~cm}^{-3}$, and the velocity corresponding to the white dwarf core temperature of $T_{\text {core }} \approx 10^{8} \mathrm{~K}$, is of the order of $\approx$ $2 \times 10^{8} \mathrm{~cm} / \mathrm{s}$, and the corresponding frequency is $v \approx 10^{18} \mathrm{~Hz}$. And for a white dwarf, $\sum \frac{d \sigma_{12}}{d \Omega} \sin ^{2} \theta \approx$ $10^{-25} \mathrm{~cm}^{2}$. The power of thermal gravitational waves emitted by the white dwarf works out to be of the order of, $\dot{E} \approx 10^{19} \mathrm{erg} / \mathrm{s}$, at a frequency of, $v \approx 10^{18} \mathrm{~Hz}$.

In the case of neutron stars, $n_{1}=n_{2} \approx 10^{38} \mathrm{~cm}^{-3}$, and the velocity corresponding to the nascent neutron star core temperature of $T_{\text {core }} \approx 5 \times 10^{10} \mathrm{~K}$, is of the order of $\approx 5 \times 10^{9} \mathrm{~cm} / \mathrm{s}$, and the corresponding frequency is $v \approx 10^{21} \mathrm{~Hz}$. And for a neutron star, $\sum \frac{d \sigma_{12}}{d \Omega} \sin ^{2} \theta \approx 10^{-29} \mathrm{~cm}^{2}$. The power of thermal gravitational waves emitted by the neutron star works out to be of the order of, $\dot{E} \approx 10^{29} \mathrm{erg} / \mathrm{s}$, at a frequency of, $v \approx 10^{21} \mathrm{~Hz}$. 
If one out of every 100 stars is a neutron star, then the power associated with the thermal gravitational waves from all the neutron stars is, $\dot{E} \approx 10^{49} \mathrm{erg} / \mathrm{s}$. The total energy emitted due to the thermal gravitational waves by these NS cores over the average life span of their nascent hot-core stage of $\sim 10^{8} \mathrm{~s}$ is, $E \approx 10^{57} \mathrm{erg}$.

\subsection{Thermal Gravitational Waves from PBHs}

Primordial black holes (PBHs) are hypothetical type of black holes that are formed not by the gravitational collapse of a star but by the extreme densities of matter present during early Universe [11]. As these PBHs evaporate through Hawking radiation, part of the energy could be released in the form of thermal gravitational waves [5]. In the case of spontaneous graviton emission, the quadrupole gravitational power is given by:

$$
\dot{E}=\frac{G}{c^{5}} m^{2} \omega^{6} R_{s}^{4}
$$

A typical PBH, that evaporates over the Hubble time has a mass of $\sim 10^{14} \mathrm{~g}$ and the corresponding Schwarzschild radius, $R_{S} \approx 10^{-13} \mathrm{~cm}$. The power due to the gravitational waves can be written as:

$$
\dot{E}=\frac{G}{c^{5}}\left(\frac{m v^{2}}{t}\right)^{2}
$$

where $v$ is the typical velocity and $t$ is the time scale, of the underlying explosive processes giving rise to the gravitational wave emission. The term within the bracket corresponds to the power of the explosion, therefore we have, $\dot{E}=\frac{G}{c^{5}} P_{\text {exp }}^{2}$. The total energy released in the form of gravitational waves is then,

$$
E=\left(\frac{G}{c^{5}} P_{\text {exp }}^{2}\right) t
$$

The number of quanta of gravitational waves is then given by:

$$
N_{G W}=\frac{N_{G W}}{\hbar / t}=\frac{G}{c^{5}} \frac{E_{\text {exp }}^{2}}{\hbar}
$$

where, $E_{\text {exp }}=P_{\text {exp }} t$ is the energy of the explosion and $\hbar / t$ is the energy of each gravitational wave quanta. For the typical PBH of mass $\sim 10^{14} \mathrm{~g}$, the energy of the explosion is:

$$
E_{\text {exp }}=m c^{2} \approx 10^{35} \mathrm{erg}
$$

And the number of quanta, $N_{G W} \approx 10^{38}$. The frequency and the corresponding energy of the gravitational wave quanta are given by, $v=1 / t$ and $\epsilon=\hbar / t$, respectively. Here $t=R_{s} / c \approx 10^{-23} \mathrm{~s}$. Therefore each quanta of gravitational waves has an energy of $\approx 10^{-4} \mathrm{erg}(100 \mathrm{MeV})$ with a frequency of $\approx 10^{23} \mathrm{~Hz}$. The total energy associated with the thermal gravitational waves is then given as, $E=$ $N_{G W} \epsilon \approx 10^{34} \mathrm{erg}$.

We see that $10 \%$ of the energy of $\mathrm{PBH}$ explosion is converted to high frequency gravitational waves, with a typical frequency of $\approx 10^{23} \mathrm{~Hz}$ corresponding to $\sim 100 \mathrm{MeV}$.

According to current estimates, the number density of PBHs in the Universe could be $\sim 1 /(k p c)^{3}$ [12]. Then the total energy associated with the gravitational waves from these PBHs over the entire volume of the Universe of $\left(10^{28} \mathrm{~cm}\right)^{3}$ is, $E_{\text {total }} \approx 10^{54} \mathrm{erg}$. This corresponds to a cosmic background energy density of $\approx 10^{-30} \mathrm{erg} / \mathrm{cc}$, and a flux of, $f_{T} \approx 10^{-20} \mathrm{erg} / \mathrm{cm}^{2} / \mathrm{s}$ in high energy thermal gravitational waves at typical energies of $\sim 100 \mathrm{MeV}$.

If the number density of $\mathrm{PBH}$ is higher, say, $\sim 1 /(p c)^{3}$, then the flux would be $f_{T} \approx$ $10^{-10} \mathrm{erg} / \mathrm{cm}^{2} / \mathrm{s}$.

The above discussion pertains to only those $\mathrm{PBH}^{\prime}$ s that evaporate over the Hubble time of $10^{10}$ years. The integrated flux from all the $\mathrm{PBH}^{\prime}$ s over the entire Hubble time will give the background thermal gravitational flux. The number of the $\mathrm{PBH}^{\prime} \mathrm{s}$ as a function of mass can be written as [13]:

$$
n_{b h}(m)=n_{b h}\left(m_{0}\right)\left(\frac{m_{0}}{m}\right)^{-n}
$$

where, $n_{b h}\left(m_{0}\right)$ is the present number density of PBHs, $m_{0}$ is the mass of the PBH that evaporate over the Hubble time and $n=3$. The integrated energy of the gravitational wave emission over the entire volume of the Universe is given by:

$$
E_{\text {total }}=\int_{m_{1}}^{m_{2}} \frac{m^{2} c^{4}}{\hbar} \frac{c}{H_{0}} n_{b h}\left(m_{0}\right)\left(\frac{m_{0}}{m}\right)^{-n} d m \times V
$$


The upper limit of the integral $m_{2}=10^{14} \mathrm{~g}$ is the mass of the PBH that will evaporate over the Hubble time scale and the lower limit $m_{1}=10^{13} \mathrm{~g}$, below which the flux is too small. Volume $=2 \pi^{2} R_{H}^{3}$, where $R_{H}=10^{28} \mathrm{~cm}$ is the Hubble radius. With these values we get the total integrated energy as, $E_{\text {total }} \approx 10^{32} \mathrm{erg}$, and the total integrated energy flux as, $f_{\text {total }} \approx 10^{-24} \mathrm{erg} / \mathrm{cm}^{2} / \mathrm{s}$.

\subsection{Thermal gravitational waves from gamma ray bursts}

Similar to stellar cores, in GRBs also, Coulomb collisions can result in the emission of high frequency gravitational waves. The power of the thermal gravitational waves is given by the same expression as that for the stars, but the bulk properties will be altered by factors of $\Gamma$ (Lorentz factor), due to the relativistic velocities encountered in GRBs. The number density will be increased by a factor of gamma and the volume associated with the GRB, will be given by the deceleration volume. In gamma ray bursts, due to the general relativistic effects, the shock wave (from a spherical shell around the blast) propagated from the burst will be decelerated.

With the Lorentz factor taken into consideration, the power of the thermal gravitational waves emitted from a GRB will be [4]:

$$
\dot{E}=\left(\frac{32 G}{5 c^{5}}\left(\Gamma \mu_{12}\right)^{2} n^{2} v_{12}^{5}\left(\frac{e^{4}}{\mu_{12}^{2} v_{12}^{4}}\right)\right)\left(\frac{E_{\gamma}}{\Gamma^{2} n m_{p} c^{2}}\right) v
$$

where $E_{\gamma} \approx 10^{51} \mathrm{erg}$, is the energy of the GRB. For $n=10^{2} \mathrm{~cm}^{-3}$, we have the power, $\dot{E} \approx$ $\Gamma^{4} \times 3.5 \times 10^{15} \mathrm{erg} / \mathrm{s}$. For a gamma ray burst corresponding to a $\Gamma=100$, the power works out to be of the order of $3.5 \times 10^{23} \mathrm{erg} / \mathrm{s}$ at the frequency of $v \approx 10^{22} \mathrm{~Hz}$.

\subsection{Thermal gravitational waves from short duration GRB}

Short duration gamma ray bursts have a shorter duration $(<0.2-2 s)$ and a harder spectrum as compared to the duration of $2-200 \mathrm{~s}$ for long GRBs. Short GRBs are due to the merger of two neutron stars, whereas, the long GRBs are due to the collapse of very massive stars. The spectrum observed is harder because the objects merging to produce the GRB are more compact.

The time taken for the merging of two NS is given by, $t_{\text {merge }}=\frac{G M^{2} / R}{\dot{E}}$. For two neutron stars of mass $1.5 M_{\odot}$ the merger time typically works out to be of the order of $10^{9}$ years. Due to the longer merger time of the NS, the short GRBs are found in older population elliptical galaxies [14].

In the case of the merger of two neutron stars, the number density as well as the temperature is substantially high compared to the long duration GRB, with temperature of the order of $10^{13} \mathrm{~K}$ and $n \approx$ $10^{40} \mathrm{~cm}^{-3}$. Considering all the gamma factors associated with the GRB $[15,16]$, as in the previous case, the gravitational power is given by, $\dot{E} \approx \Gamma^{4} \times 4 \times 10^{43} \mathrm{erg}$, at a frequency of $v \approx \Gamma \times 10^{23} \mathrm{~Hz}$.

During the short duration burst, the two neutron stars undergo collision. During the tidal breakup of the neutron stars, its binding energy is released, which for a pair of neutron stars each of mass $1.5 M_{\odot}$ and radius of about $10^{6} \mathrm{~cm}$, is

$$
B E=2\left(\frac{3}{5} \frac{G M^{2}}{R}\right) \approx 6 \times 10^{53} \mathrm{erg}
$$

For a gamma factor of about 100 , the power radiated due to the thermal gravitational wave emission is of the order of $\dot{E} \approx 4 \times 10^{51} \mathrm{erg} / \mathrm{s}$. This implies that about $1 \%$ of the energy released in the short duration gamma ray burst could be in the form of thermal gravitational waves. For a GRB at a typical distance of $100 \mathrm{Mpc}$, the flux is given by:

$$
f=\frac{E}{4 \pi d^{2}} \approx 4 \times 10^{-3} \mathrm{erg} / \mathrm{s} / \mathrm{cm}^{2}
$$

Since the event occurs over a time scale of one second, the flux is equivalent to the fluence.

\section{Cosmic background thermal gravitational radiation}

At the Planck epoch in the early Universe, with $t_{P l} \approx 10^{-43} s$, all the interactions were of equal strength so that thermal equilibrium was maintained between gravitons and other particles. As the Universe expanded, the gravitational interaction weakened and gravitons decoupled from other 
particles [9]. If $N$ is the number of particles that were coupled with the graviton, then the temperature of the background thermal gravitational radiation is given by:

$$
T_{g}=\left(\frac{43}{22 N}\right)^{1 / 3} T_{r}
$$

where, $T_{r}$ is the background radiation temperature. And for the present cosmic radiation temperature of $2.7 \mathrm{~K}$ and with $N \approx 30$, we have the temperature of the background thermal gravitational radiation of about $1 K$.

This result has consequences for the inflationary model of the Universe. At the time of inflation, the expansion of the Universe occurred at an exponential rate where the expansion was increased by a factor of $10^{28}$, when the Universe was $10^{-36} s$ old [17]. The radiation temperature at this epoch is given by:

$$
T=\frac{2 \times 10^{10}}{\sqrt{t}} \approx 2 \times 10^{28} \mathrm{~K}
$$

The temperature of the background thermal gravitational radiation corresponding to this radiation temperature is given by,

and the corresponding wavelength is,

$$
T_{g}=\left(\frac{43}{22 N}\right)^{1 / 3} T_{r} \approx 8 \times 10^{27} \mathrm{~K}
$$

$$
\lambda=\frac{\hbar c}{k T_{g}} \approx 10^{-28} \mathrm{~cm}
$$

At the end of the inflation phase, the wavelength of the gravitational radiation background is $1 \mathrm{~cm}$. However, in order not to interfere with nucleosynthesis in the hot dense phase, its energy density would have to be $<1 \%$ of the radiation energy density. This would give an $h$ of the order of $\frac{\hbar^{2} \omega^{2} c^{2}}{G}$. Now the wave would be stretched by a further factor of $10^{19}$ (at the present epoch). This would give a wavelength of $10^{19} \mathrm{~cm}$ at present. Detection of such waves through fluctuations in the cosmic microwave background radiation could verify the existence of such a phase in the early Universe [18]. If inflation had not taken place, we would be left with a thermal gravitational wave background with a temperature of $\sim 1 K$. If at all this can be detected, it would provide evidence against inflation [7].

Also the detection of these thermal background gravitational waves provides a basis to verify the validity of the big bang model itself. There are claims that the most convincing evidences of the big bang, the microwave background and abundance of helium, can be accounted for without invoking the big bang. But these thermal gravitational waves cannot be generated without the Universe passing through the super-hot, super-dense Planck epoch [19].

\subsection{Integrated background thermal gravitational radiation}

Thermal gravitational waves continue to be generated in the early Universe as it expands, as the temperature and particle densities continue to be high. We can estimate the integrated power emitted in thermal gravitational waves as the Universe cools from say $10^{13} \mathrm{~K}-10^{5} \mathrm{~K}$ [20]. This is similar to what was done for stellar core (in section 3.1) but now all the quantities are time dependant. The time range, corresponding to the above temperatures is $10^{-6} s-10^{10} s$. The power per unit volume per unit frequency interval is given by the same expression as in equation (13).

The number density is dependent on time as, $n=n_{i}\left(\frac{t_{i}}{t}\right)^{2}$, where the quantities with subscript $i$ indicates the initial values of theses quantities at time of $10^{-6} \mathrm{~s}$. The time dependence of frequency and velocity are, $v=v_{i}\left(\frac{t_{i}}{t}\right)^{1 / 2}$ and $v=v_{i}\left(\frac{t_{i}}{t}\right)^{1 / 4}$, respectively. Since the radius of the Universe is related to the temperature as, $R T=$ constant, the volume is given as, $V=V_{i}\left(\frac{t}{t_{i}}\right)^{3 / 4}$. Hence the power radiated by the thermal background gravitational radiation is:

The initial values corresponding to time $10^{-6} S$ is given by:

$$
\dot{E}=\left(\frac{32 G}{5 c^{5}}\right) n_{i}^{2} v_{i} e^{4} V_{i} v_{i}\left(\frac{t}{t_{i}}\right)^{3 / 4}
$$

$$
n_{i}=n\left(\frac{t}{t_{i}}\right)^{2}=1 \text { proton } / m^{3}\left(\frac{13.7 \times 10^{9} \times 3.15 \times 10^{7}}{10^{-6}}\right)^{2}
$$


$v_{i}=\frac{k T_{i}}{h} \approx 2 \times 10^{23} \mathrm{~Hz} ; v_{i}=\sqrt{\frac{3 k T_{i}}{m_{p}}} \approx c ; V_{i}=2 \pi^{2} R_{i}^{3} \approx 10^{48} \mathrm{~m}^{3}$

This gives the power as, $\dot{E}=\frac{2 \times 10^{56}}{t^{3 / 4}}$. The integrated energy (over the time range of $10^{-6} s-10^{10} s$ ) is given by:

$$
\int \dot{E} d t=\int_{10^{-6}}^{10^{10}} \frac{2 \times 10^{56}}{t^{3 / 4}} d t \approx 10^{67} \text { erg }
$$

This is the integrated energy radiated by the background thermal gravitational radiation in the early Universe as the temperature cooled from $10^{13} \mathrm{~K}-10^{5} \mathrm{~K}$, and this is spread over the Hubble volume.

As the Universe expanded, this energy is red shifted by a factor given by, $\frac{R_{\text {present }}}{R_{\text {at }} 10^{5} \mathrm{~K}} \approx 10^{5}$. Therefore the present energy associated with the integrated background thermal gravitational radiation is of the order of $10^{62} \mathrm{erg}$. The flux associated with this integrated background thermal gravitational radiation (IBTGR) is given by, $f \approx 2 \times 10^{-14} \mathrm{erg} / \mathrm{s} / \mathrm{cm}^{2}$.

The energy density associated with the cosmic microwave background radiation is of the order of $10^{-13} \mathrm{erg} / \mathrm{cm}^{3}$, and the corresponding flux is, $f_{C M B} \approx 10^{-3} \mathrm{erg} / \mathrm{s} / \mathrm{cm}^{2}$. We see that the flux associated with integrated background thermal gravitational radiation is about 10 orders less than that of the $\mathrm{CMB}$ radiation.

The contribution of the microwave background radiation to the normalised critical density of the Universe is $\Omega_{C M B} \approx 4 \times 10^{-5}$. Since the flux associated with IBTGR is about 10 orders less the contribution to $\Omega$ due to this will be $\Omega_{I B T G R} \approx 10^{-15}$. The overall energy associated with the emission of thermal gravitational waves from stellar sources is of the order of $10^{57} \mathrm{erg}$, which is still about 5 orders less than that associated with IBTGR $\left(10^{62} \mathrm{erg}\right)$ as arrived at earlier.

As for the PBH's, we need something like $10^{30}$ of them to match the thermal gravitational wave background. This would need a density of such objects much more than what is implied by the gamma ray background.

\section{Detection of thermal gravitational waves}

When a weak gravitational wave passes through a system of particles along a line perpendicular to the plane of the particles then the particles will oscillate. The area enclosed by the particles does not change, and there is no motion along the direction of propagation. Passing of gravitational waves through a system of masses sets it into harmonic oscillations, hence causing a strain $h$.

However, current devices in operation like LIGO are expected to detect an $h \approx 10^{-22}$. But what about the detection of thermal gravitational waves. So far there have been few attempts to conceive detection of such waves. The flux of thermal gravitational wave from the Sun (around frequency of $\approx$ $\left.10^{16} \mathrm{~Hz}\right)$ at earth is about $0.5 \mathrm{~W}\left(5 \times 10^{6} \mathrm{erg}\right)$. How can we detect this kind of high frequency gravitational wave radiation? For example, in magnetised plasma, gravitational waves can be coupled to electromagnetic waves and can get damped. The damping time is given by:

$$
\tau=\frac{\omega_{B}}{G n T^{1 / 2} m_{n}^{3 / 2}}
$$

For a magnetic field of $10^{15} \mathrm{G}$ and $T \approx 10^{12} \mathrm{~K}$, the damping time is of the order of $10^{2} \mathrm{~s}$.

If two particles in the gas is separated by a distance of $d$, and the passing of gravitational wave causes a strain $h$, then the change in the distance between the particles is given by, $\Delta d=h d$. The distance of separation is given by, $d=v / \omega$ where, $\omega$ is the frequency and $v=\sqrt{\mathrm{kT} / \mathrm{m}}$ is the particle velocity. The change in velocity due to the passing of the gravitational wave is given by, $\Delta v=\hbar d \omega$, and the energy change per collision is given by, $d E=\frac{1}{2} m\left(\frac{\Delta v}{v}\right)^{2}$. If the number density of particles is $n$ and $\lambda$ is the mean free path, the total power radiated over the volume is given as:

$$
\dot{E}=\frac{1}{2} m\left(\frac{\Delta v}{v}\right)^{2} \lambda n=\frac{1}{2} m^{2} \lambda n \frac{(\hbar d \omega)^{2}}{k T}
$$

The energy density of the wave is of the order of, $\frac{(\hbar \omega c)^{2}}{G}$. On integrating the expression for power we get the time scale for the damping of the wave as [4]:

$$
\tau=\lambda\left(\frac{c}{v}\right)^{3}\left(\frac{\omega^{2}}{G m n}\right)=\lambda\left(\frac{c}{v}\right)^{3}\left(\frac{\omega^{2}}{\omega_{G}^{2}}\right)
$$


The quantity $G m n$ has the same dimensions as $\omega^{2}$, hence $\sqrt{G m n}$ can be interpreted as the gravitational plasma frequency $\omega_{G}$ associated with the particles undergoing oscillations due to the passing of the gravitational waves.

In the case of neutron stars with temperature of the order of $T \approx 10^{11} \mathrm{~K}$ we have $\omega \approx \omega_{G}$. The damping time will be of the order of $10^{-18} \mathrm{~s}$ and hence waves may be trapped within the NS. The trajectories of charged particles may be affected by passage of gravitational waves, which involves generation of electric current in the magnetised plasma.

The high frequency gravitational waves can also be detected through the atomic transitions induced by them at a very slow rate [7]. The quadrupole transition of hydrogen from $3 d \rightarrow 1 s$ state with emission of a graviton occurs at a frequency of $\approx 10^{15} \mathrm{~Hz}$. The quantum mechanical transition rate is given by [21]: $\zeta=\frac{P}{\hbar \omega}$, where, $P$ is the power emitted by a dipole. For the case of spontaneous graviton emission, the quadrupole gravitational power is given by, $P=\frac{2 G \omega^{6}}{5 c^{5}} I^{2}$. Therefore we have the transition rate:

$$
\zeta=\frac{P}{\hbar \omega}=\frac{\alpha^{2} G m_{e}^{3} c}{360 \hbar^{2}} \approx 6 \times 10^{-40} s^{-1}
$$

where, $\alpha=\frac{\hbar}{m_{e} e^{2}}$ is the Bohr radius. And the corresponding lifetime of the transition is:

$$
\tau=\frac{5 \hbar^{2}}{256 G c \alpha^{6} m_{e}^{3}} \approx 10^{36} S
$$

The frequency of this transition is of the order of $10^{16} \mathrm{~Hz}$, which is within the range of thermal gravitons emitted from the Sun. About $10^{3}$ gravitons fall per square metre per second on the Earth from the Sun at this frequency. Thus there is a finite probability of detecting induced emission with a sufficiently large detector. This radiation will be very penetrating.

By coincidence, the lifetime for this transition to take place is the same as the proton decay time $\left(10^{38} \mathrm{~s}\right)$. Proton decay is a major prediction of GUTs and despite the long lifetime is being tested by several experiments. So it may not altogether be impossible to also observe the effects of high frequency thermal gravitational radiation, which can also induce transitions with a lifetime comparable to that of proton decay.

The absorption rate of these high frequency gravitons can be estimated as $\approx 10^{-27}$, for terrestrial detectors, so one requires a detector of several hundred square kilometres to detect a few transitions in some decades [22].

Another way of detecting these thermal GW is to convert them into electromagnetic waves of same frequency. When an electromagnetic wave of amplitude $H_{Y}$ propagates through a constant magnetic field $H_{0}$, as it produces a quadrupole stress term given by:

$$
T_{Y Y}=H_{Y} H_{0} \cos (k x-\omega t)
$$

This stress term gives rise to gravitational waves given by the linear Einstein equation [20]:

$$
\square h_{Y Y}=k T_{Y Y}, k=\frac{16 \pi G}{c^{4}}
$$

Alternatively, a weak gravitational wave $h_{Y Y}$ propagating through a magnetic field $H_{0}$ gives rise to a magnetic field perturbation given by [23]:

$$
\square H_{Y}=\omega^{2} h_{Y Y} H_{0}
$$

The fraction of the gravitational wave energy converted into electromagnetic waves of frequency $\omega$ is given by [24,25]: $f=k H_{0}^{2} d^{2}$, where, $d$ is the spatial extent of the uniform magnetic field.

\section{Conclusions}

The gravitational waves detected so far by the LIGO and Virgo collaboration are from transient events, such as the final stages of mergers of black holes and neutron stars. These waves have frequencies in a band ranging from a few hundred hertz to around a kilohertz to which LIGO type instruments are sensitive. Here we study various sources of continuous background gravitational waves. The frequency and background flux for a whole mass range of compact binary and isolated compact objects are estimated. As shown, these gravitational wave backgrounds are produced in typical frequency ranges, over a Hubble time. The fluctuations in the strain due to these background GW fall within the sensitivity range of either existing or proposed GW detectors. 
Such gravitational waves are also associated with close planetary stellar entities. For instance the total power emitted in GW by Jupiter as it orbits the Sun is about a few kilowatts. For a system of a hot Jupiter $\left(10 M_{J}\right)$ orbiting a solar mass star with a period of a day (at a distance of $\approx 0.02 \mathrm{AU}$ ) the power emitted in GW is $\approx 7 \times 10^{24} \mathrm{erg} / \mathrm{s}$, at a frequency of $10^{-5} \mathrm{~Hz}$. There could also be new class of planetary dark matter objects that could emit gravitational radiation [26].

We have also given detailed estimates of the high frequency thermal gravitational radiation flux emitted from a variety of high energy sources including nascent neutron stars, gamma ray bursts, evaporating primordial black holes, etc. We have also estimated the integrated thermal gravitational wave flux produced in the expanding early universe. This integrated cosmic thermal gravitational wave background has a present energy density about five orders of magnitude higher than that from all other discrete sources. Gravitational waves also provides a testing tool for various theories of gravity, i.e. general relativity and extended theories of gravity, and will be the definitive test for general relativity [27].

\section{References}

1. Abbott, B.P. et al. Observation of Gravitational Waves from a Binary Black Hole Merger. Phys. Rev. Lett. 2016, $116,061102$.

2. Abbott, B.P. et al. Multi-messenger Observations of a Binary Neutron Star Merger. Astrophys. J. 2017, 848, L12.

3. Amaro-Seoane, P. et al. Low-frequency gravitational-wave science with eLISA/NGO. Class. Quantum Grav. 2012, 29, 124016.

4. Sivaram, C.; Arun, K. Thermal Gravitational Waves. Open Astron. J. 2011, 4 (Suppl 1-M2), 65. arXiv:0708.3343, 24 August 2007.

5. Sivaram, C.; Arun, K. Thermal Gravitational Waves from Primordial Black Holes. Open Astron. J. 2011, 4 (Suppl 1-M3), 72. arXiv:1005.3431, 19 May 2010.

6. Sartore, N.; Ripamonti, E.; Treves, A.; Turolla, R. Galactic neutron stars - I. Space and velocity distributions in the disk and in the halo. Astron. Astrophys. 2010, 510, A23.

7. Sivaram, C. Thermal gravitational radiation from stellar objects and its possible detection. Bull. Astron. Soc. India 1984, 12, 350.

8. Sivaram, C. OJ 287: New Testing Ground for General Relativity and Beyond. arXiv:0803.2077 2008.

9. Weinberg, S. Gravitation and Cosmology; Wiley: New York, USA, 1972.

10. Cox, J.P; Guili, R.T. Principles of Stellar Structure; Pergamon: New York, USA, 1968.

11. Sivaram, C.; Arun, K. Charged Black Holes and Constraints on Baryon Asymmetry. arXiv:1003.1667 2010.

12. Montero-Camacho, P. et al. Revisiting constraints on asteroid-mass primordial black holes as dark matter candidates. J. Cosmol. Astropart. Phys. 2019, 08, 031.

13. Shapiro, S.L.; Teukolsky, S. White Dwarfs, Neutron Stars and Black Holes; Wiley: New York, USA, 1983.

14. Chincarini, G. Gamma-Ray Bursts: Learning about the Birth of Black Holes and Opening new Frontiers for Cosmology. Messenger 2006, 123, 54.

15. Sivaram, C. Constraints on the photon mass and charge and test of equivalence principle from GRB 990123. Bull. Astron. Soc. India 1999, 27, 627.

16. Tokuoka, T. Interaction of Electromagnetic and Gravitational Waves in the Weak and Short Wave Limit. Prog. Theor. Phys. 1975, 54, 1309.

17. Zel'dovich, Ya.B; Novikov, I.D. Relativistic Astrophysics Vol. II.; Univ. of Chicago Press: Chicago, USA, 1997.

18. Zel'dovich, Ya.B. Primordial Magnetic Fields and Gravitational Wave Effects on CMBR. Soviet Physics JETP $1973,38,652$.

19. Biscoveanu, S.; Talbot, C.; Thrane, E.; Smith, R. Measuring the Primordial Gravitational-Wave Background in the Presence of Astrophysical Foregrounds. Phys. Rev. Lett. 2020, 125, 241101.

20. Abbott, B., et al. Upper limits on gravitational wave bursts in LIGO's second science run. Phys. Rev. D 2005, $72,062001$.

21. Misner, C.W; Thorne, K.S.; Wheeler, J.A. Gravitation, Freeman: San Francisco, USA, 1973.

22. De Logi, W.K, Mickelson, A.R. Electrogravitational conversion cross sections in static electromagnetic fields. Phys. Rev. D 1977, 16, 2915. 
23. Sivaram, C. Plasma Damping of Gravitational Waves. Proceedings of the IAU Symposium No. 142, Bangalore, India, 1-5 December 1989; E. Priest, V. Krishnan, Eds. Kluwer Academic Press: Boston, USA 1990.

24. Boughn, S.; Rothman, T. Aspects of graviton detection: graviton emission and absorption by atomic hydrogen. Class. Quantum Grav. 2006, 23, 5839.

25. Chen, P. Gravitational Beamstrahlung. Mod. Phys. Lett. A 1991, 6, 1069.

26. Sivaram, C.; Arun, K. Dark Matter Objects: Possible New Source of Gravitational Waves. Earth Moon Planets 2019, 123, 9 .

27. Corda, C. Interferometric Detection of Gravitational Waves: the Definitive Test for General Relativity. Int. J. Mod. Phys. D 2009, 18, 2275. 